\title{
Uncommon cause of small bowel obstruction - gallstone ileus: a
} case report

\author{
Fatima Ezzahra Zahid*, El Bachir Benjelloun, Abdelmalek Ousadden, \\ Khalid Mazaz and Khalid Ait Taleb
}

Address: Department of general surgery, University hospital Hassan II, Fes, Morocco

Email: Fatima Ezzahra Zahid* - fatizahid78@hotmail.com; El Bachir Benjelloun - benjelloun19@hotmail.com; Abdelmalek Ousadden - aousadden@yahoo.fr; Khalid Mazaz - khalid.mazaz@caramail.com; Khalid Ait Taleb - chufes@menara.ma

* Corresponding author

Published: 14 December 2009

Cases Journal 2009, 2:932I doi:10.1186/I757-1626-2-932I

This article is available from: http://www.casesjournal.com/content/2/I/932I

(C) 2009 Zahid et al; licensee BioMed Central Ltd.

This is an Open Access article distributed under the terms of the Creative Commons Attribution License (http://creativecommons.org/licenses/by/2.0), which permits unrestricted use, distribution, and reproduction in any medium, provided the original work is properly cited.

\section{Abstract}

Gallstone ileus is an uncommon cause of small bowel obstruction. We present a case of small intestinal obstruction owing to a large gallstone in lower ileum in a 65 years old man. The diagnosis was made by computed tomography.
Received: 31 December 2008

Accepted: 14 December 2009

\section{Background}

Gallstone ileus is an uncommon condition that may result when a gallbladder stone enters into the intestinal tract, usually as a result of an internal fistula between the gallbladder and the duodenum. It's accounting for only 1$4 \%$ of all intestinal obstruction [1]. In patient with cholilithiasis only $0.3-0.5 \%$ develop gallstone ileus [2]. The mortality reportedly ranges from $12-18 \%$, particularly in older patient who often have co morbid illness [3]. We reported a case of a 65 years old man, who present with signs of small bowel obstruction, owing to a large gallstone in lower ileum.

\section{Case Presentation}

A 65-year old previously healthy man, presented to the Emergency Department, with complaints of abdominal pain, vomiting and absolute constipation of 2 days duration. The patient was not using any specific medication and his medical history did not suggest a major disease. He had no prior history of abdominal surgery or trauma. The patient didn't smoke or drink alcohol. 


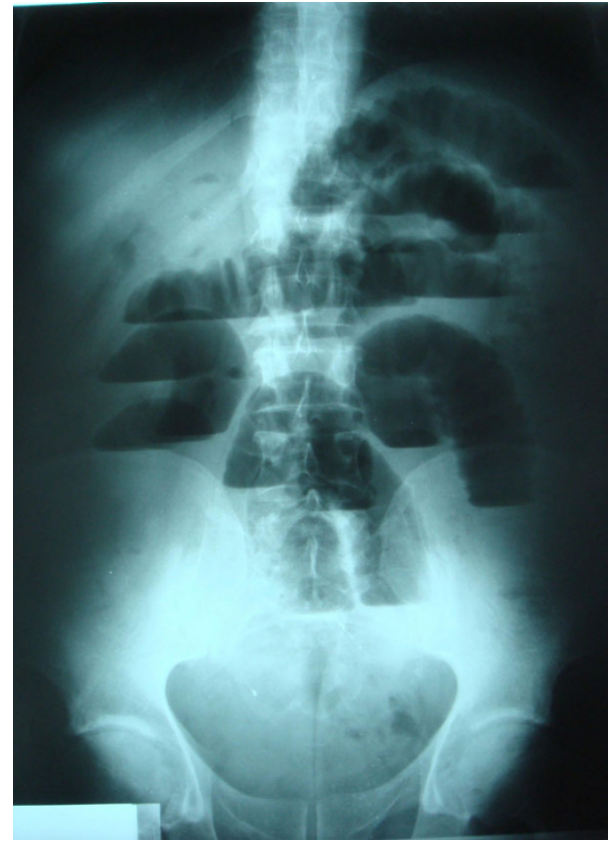

Figure I

Abdominal $X$ ray shows air fluid levels.

ileum $3 \mathrm{~cm}$ before Bauhin valve (fig 3). The gallstone was extracted by means of a longitudinal enterotomy, which was then closed transversally. The gallbladder was surrounded by an intense inflammatory reaction. The plan is for the patient to return for cholecystectomy and fistula repair at a later date. The follow up was simple.

\section{Discussion}

Gallstone ileus is an uncommon cause of small bowel obstruction [1-6]. It occurs almost exclusively in the eld-

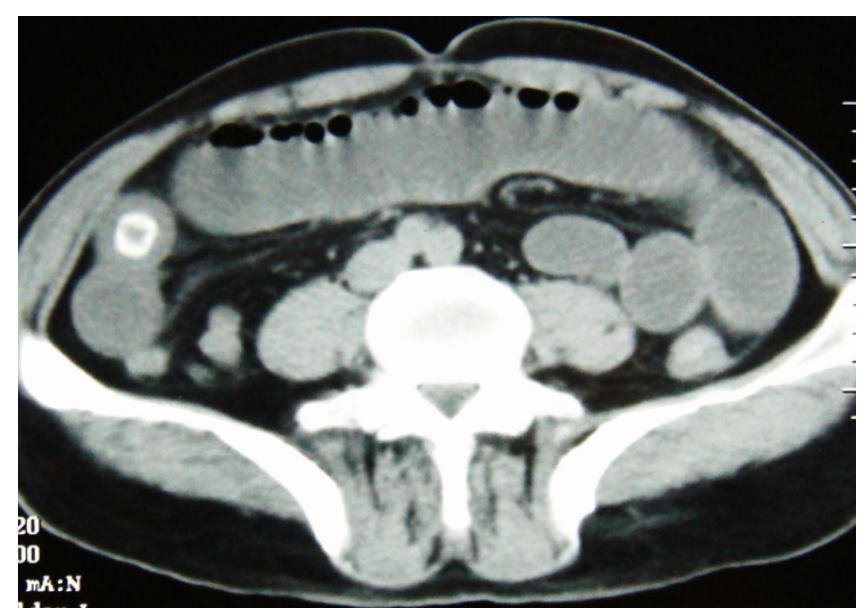

Figure 2

Abdominal tomography shows a calcified mass in lower ileum.

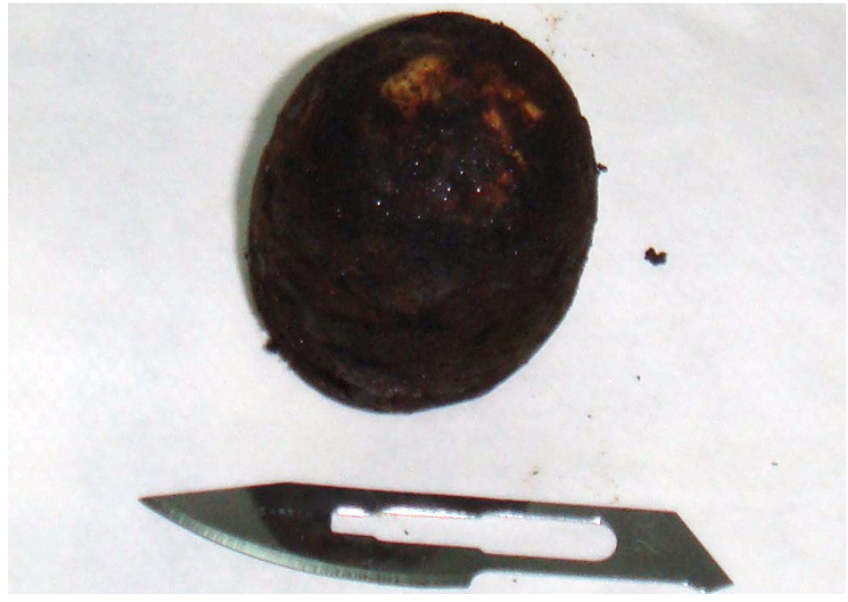

Figure 3

Gallstone extracted from the ileum.

erly, and account for $25 \%$ of mechanical small-bowel obstruction in patient over the age of 65 , with a mortality of $12-50 \%$ [4]. This pathology occurs three to five times more frequently in women than in men [2]. The gallstone enters the intestinal tract through a fistula formed between the gallbladder and the duodenum, stomach or colon. In particular, a cholecystodudenal fistula was identified in $68 \%$ of patients with gallstone ileus [6]. The terminal ileum is the most frequent site of obstruction [1]. However, it may be found in the duodenum causing Bouveret's syndrome [2]. Other obstruction points, including jejunum (30\%) and colon (2.5\%) may be seen. Plain abdominal radiographs may reveal signs of small bowel obstruction and concomitant aerobilia to suggest the diagnosis [7,8]. The classical radiologic triad or Rigler triad of pneumobilia, small bowel obstruction and ectopic gallstone is specific for this disease, but only 9$14 \%$ of patients have a clear-cut Rigler's triad [7]. Computed tomography is the investigation of choice. The principal goal in management of gallstone ileus is a quick effective relief of mechanical bowel obstruction. Spontaneous passage of gallstones large enough to cause impaction has been reported, but most patients require intervention. If the stone is in within reach of an endoscope, either in the proximal small bowel or in the colon, it may be treated by lithotripsy and removal of the fragment[2]. Extracorporeal shockwave lithotripsy has also been used successfully, but this method is limitedby bowel gas. Unfortunately, the majority of patients require surgery. Surgical options include enterotomy and removal of the stones (enterolithotomy), enterolithotomy plus cholecystectomy and repair of the fistula [3]. Most authors favor enterolithotomy alone, followed by cholecystectomy at later date, because of its lower morbidity and report high spontaneous fistula closure up to 50\% [7]. 


\section{Conclusion}

Although it's a rare cause of bowel obstruction, gallstone ileus should be capped in mind when dealing with a case of small bowel obstruction, especially in elderly patient in whom the diagnosis is easily ignored. Early surgical intervention is the mainstay of treatment.

\section{Consent}

Written informed consent was obtained for publication of this case report and accompanying images. A copy of the written consent is available for review by the Editor-inChief of this journal.

\section{Competing interests}

The authors declare that they have no competing interests.

\section{Authors' contributions}

FZ is a surgeon who was drafting the manuscript and revising it critically for content. EB is a surgeon who was involved in literature research. KM, KA were surgeons treating of the patient and were involved in revising the draft critically for content. AO is a surgeon was getting photographs and was involved in drafting manuscript. All authors have given final approval of the revision to be published.

\section{References}

I. Chatterjee S, Tamonas C, Goutan G, Ambar G: Gallstone ileus an atypical presentation and unusual location. Int J Surj 2008, 6(6):e55-e56.

2. Chen-Wang C, Shou-Chuan S, Shee-Chau L, Cheng-Hsin C: Gallstone ileus: a disease easily ignored in the elderly. Int J Gast enterology 2008, 2(I): I8-2I.

3. Deepak J, Ali V, Tom MR, Fox C, Arun D: Bouveret's syndrome as an unusual cause of gastric outlet obstruction: a case report. Journal of Medical Case Reports 2007, I:73.

4. De Palma GD, Mastrobuni G, Benassai G: Endoscopic removal of a gallstone obstructing the lower ileum. Dig Liv Dis 2009, 4 I(6):446.

5. Ishikura H, Sakata A, Kimura S, Okitsu H, et al.: Gallstone ileus of the colon. Surgery 2005, I38:3.

6. Williams J B, Mehta S G, Vu T, Wonderlich D A: Gallstone ileus. The journal of emergency medicine . doi: I0.1016/J.Jemer.I I.063

7. Elabsi M, Amraoui M, Errougani A, Chkof MR: Diagnosis and treatment: gallstone ileus. Digestive and liver disease 2007, 39:|80-|8|.

8. Strauss M, Jacqunod P, Sezeur A, Houdart R, Ziza JM: Un syndrome occlusif. La revue de médecine interne 2009, 30(I):69-70.
Publish with Biomed Central and every scientist can read your work free of charge

"BioMed Central will be the most significant development for disseminating the results of biomedical research in our lifetime. " Sir Paul Nurse, Cancer Research UK

Your research papers will be:

- available free of charge to the entire biomedical community

- peer reviewed and published immediately upon acceptance

- cited in PubMed and archived on PubMed Central

- yours - you keep the copyright

Submit your manuscript here:

http://www.biomedcentral.com/info/publishing_adv.asp
BioMedcentral 